\title{
On the accuracy of the finite difference method for applications in beam propagating techniques
}

\author{
H.J.W.M. Hoekstra, G.J.M. Krijnen and P.V. Lambeck \\ MESA-Research Institute, University of Twente, P.O. Box 217, 7500 AE Enschede, The Netherlands
}

Received 20 July 1992; revised manuscript received 1 September 1992

\begin{abstract}
In this paper it is shown that the inaccuracy in the beam propagation method based on the finite difference scheme, introduced by the use of the slowly varying envelope approximation, can be overcome in an effective way. By the introduction of a perturbation expansion the accuracy can be improved as much as wanted, often nearly without any increase of the computation time. An error analysis is given and the method is illustrated by an example.
\end{abstract}

\section{Introduction}

Among the various beam propagation methods (BPM) used for simulations in integrated optics, the finite difference method (FDM) is one of the most general applicable algorithms [1-3]. Using the slowly varying envelope approximation (SVEA), the computation time can be greatly reduced. However, this approximation causes considerable errors if the effective indices of the various modes involved differ too much [4]. In this paper we aim to show that these errors can be overcome to any extend in a simple way without much increase in computation time.

\section{Theory}

\subsection{Zeroth-order approximation}

In this paper we will treat the $2 \mathrm{D}$ case using the scalar approximation. The conclusions, however, are quite general, and hold also in 3D for both the scalar and vectorial case. Using the SVEA the field propagating along the positive $z$-direction can be written as

$E(x, z) \equiv \psi(x, z) \exp \left(-\mathrm{i} k_{0} n_{0} z\right)$.

A time dependence $\exp (\mathrm{i} \omega t)$ is assumed and $n_{0}$ is a suitably chosen mean refractive index. Substituting eq. (1) into the wave equation it follows [1-3]:

$\mathrm{i} a \frac{\partial \psi}{\partial z}=O \psi+\frac{\partial^{2} \psi}{\partial z^{2}}$.

Here $a=2 k_{0} n_{0}$ and $O$ is an operator, $O \equiv \partial^{2} / \partial x^{2}$ $\left.+k_{0}^{2}\left[n^{2}(x, z)-n_{0}^{2}\right)\right]$. Introducing a discretisation, $\psi_{j}^{s}=\psi(s \Delta z, j \Delta x)$ with $j=0,1, \ldots, N$, eq. (2) can be written as a matrix equation

$\mathrm{i} a \frac{\partial \psi}{\partial z}=\mathbf{M} \psi+\frac{\partial^{2} \psi}{\partial z^{2}}$,

where $\mathbf{M}$ is a tridiagonal matrix and the nondiagonal matrix elements are all equal to $1 / \Delta x^{2}$ and $M_{j j}=-2 / \Delta x^{2}+k_{0}^{2}\left(n_{j}^{2}-n_{0}^{2}\right)$.

In fact, eq. (3) corresponds to eq. (2) only if the fields at the boundaries are put equal to zero. However, to suppress unwanted reflections from the boundaries it is better to use transparent boundary conditions [5], which is rather straightforward and not described in this paper.

Integrating eq. (3), using the Crank-Nicolson (CN) scheme, it follows:

$$
\begin{aligned}
& \mathrm{i} a\left(\boldsymbol{\psi}^{s+1}-\boldsymbol{\psi}^{s}\right) \\
& \quad=\mathbf{M}\left(\boldsymbol{\psi}^{s+1}+\boldsymbol{\psi}^{s}\right) \Delta z / 2+\frac{\partial \psi^{s+1}}{\partial z}-\frac{\partial \psi^{s}}{\partial z} .
\end{aligned}
$$

In the SVEA the last two terms at the rhs are usually neglected [1-4]. Then, the resulting matrix equation 
$\mathbf{R}_{+} \boldsymbol{\psi}^{s+1}=\mathbf{R}_{-} \boldsymbol{\psi}^{s}, \quad$ (zeroth-order),

with $\mathbf{R}_{+/-}=\mathbf{I} \pm \mathrm{i} \mathbf{M} \Delta z / 2 a$, can be solved in a standard fashion. Here $I$ is the identity matrix.

In order to estimate the error that is introduced this way, we assume that the propagating, discretesized field, $\psi$, consists of a single mode and that the corresponding effective index is given by $n_{\mathrm{e}}$. Then, by noting that the modal field equation is given by $O_{\mathrm{e}} \psi=0$, with operator $O_{\mathrm{e}} \equiv \partial^{2} / \partial x^{2}+k_{0}^{2}\left[n^{2}(x, z)\right.$ $\left.-n_{\mathrm{e}}^{2}\right]$, it follows:

$\mathbf{M} \boldsymbol{\psi}^{s} \equiv k_{0}^{2}\left(n_{\mathrm{e}}^{2}-n_{0}^{2}\right) \boldsymbol{\psi}^{s}$,

whence, also using eq. (5)

$\boldsymbol{\psi}^{s+1}=b_{-} \boldsymbol{\psi}^{s} / b_{+}$,

with $b_{+/-}=1 \pm \mathrm{i} k^{\prime} f, k^{\prime} \equiv k_{0} n_{0} \Delta z$ and $f \equiv\left(n_{\mathrm{e}}^{2}-n_{0}^{2}\right) /$ $\left(2 n_{0}\right)^{2}$. Note that eq. (7) has to be compared with the correct propagation of the envelope:

$\boldsymbol{\psi}^{s+1}=\boldsymbol{\psi}^{s} \exp \left[-\mathrm{i} k_{0}\left(n_{\mathrm{e}}-n_{0}\right) \Delta z\right]$.

Assuming that all the refractive indices are real, the error of the effective index, $\Delta n_{\mathrm{e}}$ can be calculated from:

$\arctan \left\{2 k^{\prime} f /\left[1-\left(k^{\prime} f\right)^{2}\right]\right\}=k^{\prime}\left(\Delta n_{\mathrm{e}}+n_{\mathrm{e}}-n_{0}\right) / n_{0}$.

Writing the arctan function as a Taylor's expansion, defining $n_{\mathrm{e}} \equiv n_{0}(1+\delta)$ and expanding the result as a series in $k^{\prime} f$, we obtain:

$\Delta n_{\mathrm{e}} / n_{0} \approx \delta^{2} / 2-2 k^{\prime 2} \delta^{3} / 3, \quad$ (zeroth-order) .

Here we have used the fact that $f=\delta / 2+\delta^{2} / 4$. From eq. (10), one may estimate the applicability of the zeroth-order approximation for a given case.

\subsection{First-order correction}

The first-order correction consists of neglecting the last two terms of eq. (4) only partially. By substitution of eq. (3), without the second derivate, into eq. (4) these terms can be taken into account approximately. Doing so we arrive at

$\mathbf{P}_{+} \boldsymbol{\psi}^{s+1}=\mathbf{P}_{-} \boldsymbol{\psi}^{s}$

with
$\mathbf{P}_{+/-}=\mathrm{I} \pm \mathrm{i} \Delta z \mathbf{M} / 2 a+\mathbf{M} / a^{2}$.

Equation (11) can be brought into the form:

$\left(\mathbf{M}+c_{1} \mathbf{I}\right) \boldsymbol{\psi}^{s+1}=c_{2}\left(\mathbf{M}+c_{3} \mathbf{I}\right) \boldsymbol{\psi}^{s}$,

and solved for $\psi^{s+1}$ as for the zeroth-order case. In eq. (12) $c_{l}(l=1-3)$ are complex constants.

In order to estimate the inaccuracy, we assume again that the propagating, discretesized field, $\psi$, consists of a single mode and that the refractive indices are real. Then,

$\boldsymbol{\psi}^{s+1}=d_{-} \boldsymbol{\psi}^{s} / d_{+}$,

with $d_{+/-}=1 \pm \mathrm{i} k^{\prime} f+f$. Proceeding as above, it follows for the inaccuracy of the effective index, $\Delta n_{\mathrm{e}}$ :

$$
\begin{aligned}
& \arctan \left\{2 k^{\prime} f(1+f) /\left[(1+f)^{2}-\left(k^{\prime} f\right)^{2}\right]\right\} \\
& \quad=k^{\prime}\left(\Delta n_{\mathrm{e}}+n_{\mathrm{e}}-n_{0}\right) / n_{0} .
\end{aligned}
$$

Whence

$\Delta n_{e} / n_{0} \approx-\delta^{3} / 4-2 k^{\prime 2} \delta^{3} / 3, \quad$ (first-order) .

Here we have collected terms up to $\delta^{3}$.

\subsection{Second-order correction}

Further improvement of the method can be obtained by substitution into eq. (4):

$$
\frac{\partial \boldsymbol{\psi}^{s}}{\partial z}=-\frac{\mathrm{i}}{a} \mathbf{M} \boldsymbol{\psi}^{s}-\frac{\mathrm{i}}{a} \frac{\partial^{2} \boldsymbol{\psi}}{\partial z^{2}} \approx-\frac{\mathrm{i}}{a} \mathbf{M} \boldsymbol{\psi}^{s}+\frac{\mathrm{i}}{a^{3}} \mathbf{M}^{2} \boldsymbol{\psi}^{s} .
$$

Here we have used eq. (3) once more for the second equality and have collected terms up to $\mathbf{M}^{2}$. As now the matrix equation to be solved contains products of the matrix $\mathbf{M}$ it is advantageous to approximate the integral $\int(-\mathrm{i} \mathbf{M} \psi / a) \mathrm{d} z$ more accurately, on integrating eq. (3). If we allow also products of the matrix $\mathbf{M}$ here, this will decrease the dependence of the error on the stepsize $\Delta z$. Using the fact that the envelope $\psi$ behaves approximately like $\exp (-\mathrm{iM} \Delta z$ ) a) it follows, in the spirit of the $\mathrm{CN}$ method:

$$
-\frac{\mathrm{i}}{a} \mathbf{M} \int_{s \Delta z}^{(s+1) \Delta z} \boldsymbol{\psi} \mathrm{d} z \approx \mathbf{R}_{-} \boldsymbol{\psi}^{s}-\mathbf{R}_{+} \boldsymbol{\psi}^{s+1},
$$




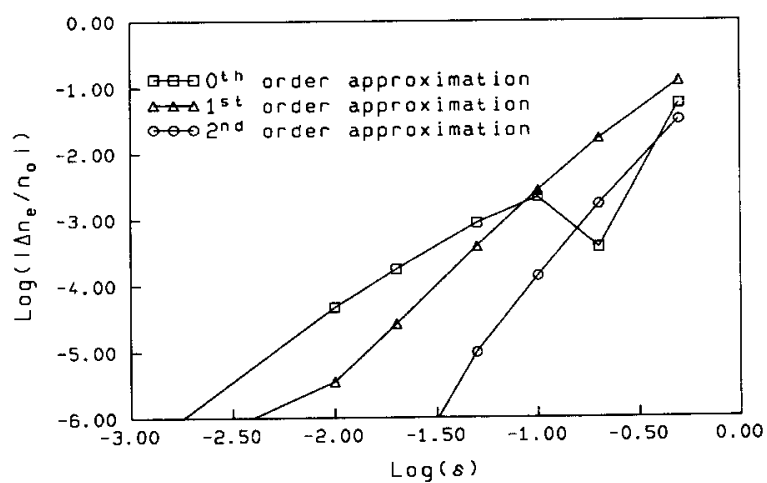

Fig. 1. $\log -\log$ plot of the relative error in the effective index with respect to the exact value as a function of $\delta$, obtained using the method outlined here (see text). The structure consists of a waveguide with a width, $w=1 \mu \mathrm{m}$, and a refractive index, $n_{\mathrm{B}}=2$, and the background index $n_{\mathrm{b}}=1$. The stepsizes and wavelength are, $\Delta z=0.5 \mu \mathrm{m}, \Delta x=0.00125 \mu \mathrm{m}$ and $\lambda=1 \mu \mathrm{m}$.

with $\mathbf{R}_{+/-} \equiv \pm \mathbf{M} \Delta z / 2 a-(\mathbf{M} \Delta z / 2 a)^{2} / 3$. Integrating eq. (3), using both eqs. (16) and (17), it follows:

$\mathbf{Q}_{+} \boldsymbol{\psi}^{s+1}=\mathbf{Q}_{-} \boldsymbol{\psi}^{s}$,

with $\mathbf{Q}_{+/-} \equiv \mathbf{I}+\mathbf{R}_{+/-}+\mathbf{M} / a^{2}-\mathbf{M}^{2} / a^{4}$.

$\psi^{s+1}$ can be solved from eq. (18) by applying two times the procedure for eq. (5), as we can write

$\mathbf{Q}_{+/-}=c_{0}\left(\mathbf{M}-c_{1,+/-} \mathbf{I}\right)\left(\mathbf{M}-c_{2,+/-} \mathbf{I}\right)$,

where $c_{l}(l=0,1,2)$ are complex constants which depend only on $n_{0}, k_{0}, \Delta x$ and $\Delta z$.

The inaccuracy of the second-order approximation can be estimated as above. Assuming again that $\psi$ is a modal field it follows:

$\psi^{s+1}=g_{-} \psi^{s} / g_{+}$, with

$g_{+/-}=1 \pm \mathrm{i} f k^{\prime}+f-f^{2}-\left(k^{\prime} f\right)^{2} / 3$.

Then,

$\Delta n_{\mathrm{e}} / n_{0} \approx \delta^{4} / 4+0.04 k^{\prime 2} \delta^{4}, \quad$ (second-order).

So, without much computational effort a considerable reduction of the error is obtained, with the help of the second-order approximation. Higher accuracy can be obtained by including higher-order terms. This can be done by simultaneously truncating eq. (16) in a later stage and better approximating the integral (17), including higher powers of $\mathbf{M}$.

We have tested all cases treated here in $2 \mathrm{D}$, with TE polarization. The results confirm the dependence of $\Delta n_{\mathrm{e}}$ on $n_{0}$ and the stepsize $\Delta z$ presented above. Results of simulations, using the formulae given above and the interface conditions according to ref. [4], are given in fig. 1 . The dip in the results of the zeroth order approximation is due to the presence of both a positive and a negative contribution to the error, according to eq. (10), which cancel for the main part at the corresponding value of $\delta$.

\section{References}

[1] D. Yevick and B. Hermansson, J. Quantum Electron. 26 (1990) 109.

[2] Y. Chung and N. Dagli, IEEE J. Quantum Electron. 26 (1990) 1335.

[3] R. Accornero, M. Artiglia, G. Coppa, P. Di Vita, G. Lapenta, M. Potenza and P. Ravetto, Electron. Lett. 26 (1990) 1959.

[4] H.J.W.M. Hoekstra, G.J.M. Krijnen and P.V. Lambeck, J. Lightwave Technol, accepted for publication.

[5] G.R. Hadley, Optics Lett. 16 (1991) 624. 